International Journal of Electrical Engineering and Technology (IJEET)

Volume 11, Issue 4, June 2020, pp. 412-421, Article ID: IJEET_11_04_046

Available online at https://iaeme.com/Home/issue/IJEET? Volume $=11 \&$ Issue $=4$

ISSN Print: 0976-6545 and ISSN Online: 0976-6553

DOI: https://doi.org/10.34218/IJEET.11.4.2020.046

(C) IAEME Publication

Scopus Indexed

\title{
EARLY MAINTENANCE AND DIAGNOSIS OF CONNECTED MACHINES USING MACHINE LEARNING
}

\section{R Srivatsan and Sharath Cherian Thomas}

Student, School of Electronics Engineering, Vellore Institute of Technology, Vellore, India

\section{Venugopal P}

Sr. Assistant Professor, School of Electronics Engineering, Vellore Institute of Technology, Vellore, India

\section{Ravi Kumar C. V}

Sr. Assistant Professor, School of Electronics Engineering, Vellore Institute of Technology, Vellore, India

\begin{abstract}
In this paper a novel system is proposed to monitor the health of industrial machines thus helping in their maintenance and early failure detection. This will help in prediction of when an industrial machine or its part will malfunction based on the data extracted from it. We will be able to replace the part or the machine in advance before any production lines get affected. This way resources will be saved and also cost of maintenance will be reduced. On top of this it is not possible to always manually monitor machines placed in remote areas like motors and pumps in water supply systems, sewage plants and chemical plants but our system would be able to not just monitor the machines but also predict the machine's current health. The proposal encompasses a lightweight machine monitoring system for next generation $M 2 M$ ecosystem for on the fly fault detection and diagnosis.
\end{abstract}

Key words: Sensors, Temperature, vibration, health prediction, microcontroller, machine learning, 5G, IoT, M2M.

Cite this Article: R Srivatsan, Sharath Cherian Thomas, Venugopal P., Ravi Kumar C.V, Early Maintenance and Diagnosis of Connected Machines Using Machine Learning. International Journal of Electrical Engineering and Technology, 11(4), 2020, pp. 412-421.

https://iaeme.com/Home/issue/IJEET?Volume $=11 \&$ Issue $=4$ 


\section{INTRODUCTION}

There are many challenges in the maintenance of machines in industries. The cost of maintenance is also quite high. Monitoring the health of the machines cannot be done always manually and thus, once the machine fails without earlier warning the production line gets affected leading to a waste of time and money [1]. This also leads to decrease in the efficiency of the process in which the machine is involved [2]. Also, manual monitoring of machines placed in inaccessible regions is very difficult and time consuming.

Modern machines need on the fly fault diagnosis to provide uninterrupted service in machine intensive and dependent scenarios such as manufacturing plants and health care units. Magnetic Resonance Imaging (MRI) technology requires heavy machine maintenance due to the innate complexity involved in the construction of the machine, It can be very crucial if such machines are allowed to operate in the non-performance regions of their performance curves due to heavy vibration or improper temperature or other factors, as this would imply incorrect diagnosis of patient's critical health conditions. Such situations require continuous and autonomous monitoring of machine health parameters. This is especially true as the machines are intensively heavy processing machines that can require large amounts of resources to run thus naturally increasing the number of parameters to be monitored for maintenance of the machine health.

Even when parameters are monitored automatically using some existing monitoring system, many systems do not give early warning by classifying the parameter values online on the fly. Such a system is not only computationally intensive but also requires a huge amount of annotated old data. The need for annotated data can be understood from the roots of the concepts of supervised learning schemes of machine learning. This puts immense pressure on the maintainer of the machine system to train the fault diagnosis models with old data for hours together. Even then there is a risk involved in the obtained model accuracy.

On the contrary to this situation, Unsupervised Learning (USL) uses the science of pattern matching to understand the difference between two sets of data. It is however still computationally intensive to use most USL algorithms for time series data that are generated often at a very high frequency of a few 100 samples per minute. This has resulted in the careful understanding and choice of the algorithm for our research and also a comparative understanding of the graphs of the results obtained from both the algorithms.

In our system various parameters of machines like temperature and vibration have been used to predict the health of the machine and the expected time before its breakdown. The health of the machine is shown to the user on his mobile using a mobile app interface. The temperature, vibration of the machine can be monitored by using various sensors and once a machine learning model has been trained, the data collected can be used to predict the condition of the machine.

\section{LITERATURE REVIEW}

A lot of knowledge has been gained over the past couple of decades about various causes for machinery problems and their effects on plant performance (Neale, 1979). Some of the major machine problems could be due to excessive vibrations, wear, worn-out bearings, damaged roller bearings, mechanical looseness, faulty belt drive, unbalanced reciprocating forces and couples, increased turbulence, electrically induced vibrations, misalignment, bent shaft, etc. (Harris and Piersol, 1996). Many researchers have conducted studies to combine the knowledge of condition monitoring with that of information technology and sensors to come up with systems for machine health monitoring for better plant performance with higher availability and reliability leading to better productivity and reduced downtime. Research on use of Artificial Intelligence (AI) techniques for machinery maintenance purposes can be 
traced to the mid 80's. The expert systems for fault diagnosis emphasized on "deep knowledge". The researchers realized the limitations of such an approach because of available knowledge which can be represented for developing expert systems (Fink and Lusth, 1987) [4]. Models were developed for condition-based health monitoring for rotating machinery using neural networks and finite-element modelling. The model integrated machinery sensor measurements through neural networks specifically trained for responding to the machine element being monitored. The proposed models had the advantage of developments in the neural networks field and finite-element-analysis. (Roemer et.al., 1996). Fuzzy models were used for residual generation and fuzzy logic for residual evaluation. The idea of "Knowledge Observer" was introduced to residual generation. (Frank and Köppen-Sliger,1997) [5]. Models were [3] developed for vibration and temperature analysis and machine condition monitoring for data interpretation, and analysis which proved to be very useful tools for both laboratory and on-site maintenance departments of large manufacturing and mineral processing plants (Frank and Köppen-Seliger, 1997, Ebersbach and Peng, 2008). The proposed methodologies aimed at optimizing maintenance cost, improving condition monitoring, health assessment and prognostics. (Labib, 1998, Smith, et.al., 2003, Rambabu, et.al., 2009) [6]. With the increase in maintenance demands, models are being developed for 24 / 7 connectivity, data security, and knowledge-sharing (Christos, et.al, 2009) [7]. As can be seen from the literature review, over the last two to three decades, a lot of research has been carried out to use the AI techniques for maintenance purposes. While some of the methodologies were for some particular application like mining, petro-chemicals, manufacturing, etc, some others were for machinery in-general.

\section{WORKING PRINCIPLE}

Industrial grade machines, for example, engines and motors produce a lot of parameters which can be used to analyze their health, whether it's working fine or malfunctioning. When a machine is running it produces heat, and there is a certain value of the heat produced by it when it is good in health and how it should vary with time [8]. If an unusual change in its value is detected, we use it to predict that it's malfunctioning. Likewise, it vibrates and also produces sound, which can be used to further confirm whether the machine is about to breakdown [9]. Thus, we have set certain standard thresholds and analyze the variation based on them. With the analysis of variation in change of data, a model was made to predict the time the machine will malfunction. The health of the machine was thus predicted and shown to the user. The temperature, vibration of the machine was recorded by using sensors and a machine learning model was trained to predict the health of the machine.

\section{IMPLEMENTATION}

With the help of 6 b\&k 4391 accelerometer sensors vibration level data will be collected and with the help of 4 Pt100 resistance temperature detectors (RTD) temperature data will be collected [14]. This data will be sent to an edge computing device by using ZigBee [15]. The edge device pre-processes the data with the help of Google Cloud IoT Edge and sends the data to cloud by making use of Google IoT core, Pub/Sub and related functions. The data is processed with the help of AI Platform. Both vibration and temperature data has been considered for demonstration of results in this paper towards the fault detection approach. A Machine Learning (DBA clustering) model has been designed to analyze the change in variation of data. With this info and standard parameters for a normal functioning machine, we could predict when the machine might face breakdown and when it may need to be replaced. The DTW algorithm is used to find the distance metric of similarity between the group of data points of vibration data of the machine over a particular time interval. This is followed by converting the vibration data at regular intervals into time series data streams. 
Once these data streams are obtained, we can divide the streams into batches of time intervals with timestamp. This data batch now becomes the dataset that is to be clustered using the distance parameter from DTW. Further, this clustering is performed using the DBA algorithm due to the lightweight nature of the algorithm in contrast to other USL approaches. This entire system consisting of the data streamlining and the k-means clustering sub-systems are incorporated into a single application that can perform the tasks continuously at intervals of 5 hours once the data collected in the stream is for instance, in the range of a few thousands. This is a good number to start clustering and predict changes in the health of the machine. Once the model has been successfully trained, it can be deployed on the edge device like an Nvidia Jetson board for detection on the edge. The predicted result can be made visible to the operators and owners via a mobile app with the help Pub/Sub and IoT Core. The IoT Edge is also capable of alerting the user in the event of an anomaly being detected. Thus, a connected reliable M2M ecosystem can be established by ensuring a mobile fault-safety methodology. In this paper code was written using python and is readily made deployable using the pickle library. Graphs are plotted using matplotlib library.

\section{MACHINE LEARNING MODEL}

The k-means clustering was used using the DTW distance metric (DBA clustering) to the time serialized input parameters- temperature /pressures of the pistons/ vibration of the gear. The results shown here in fig1. are chiefly for the vibration data due to the reduced complexity of interpretation of the results of vibration. Similar tests have been performed by running the algorithm in other datasets too.

K-means algorithm was implemented as follows:

- Value of the number of clusters was decided on i.e., $\mathrm{k}=2$ in this case (faulty and healthy).

- The cluster centroids for the $\mathrm{k}=2$ clusters are randomly initialised.

- Assigning each object to the correct cluster centroids is done by computing the DTW distance between each particular object and the centroids then optimising by finding the nearest centroid to that data point from the serialized array.

- Computation of the newly formed cluster centroids by tallying the mean of all data points present in each cluster.

- If there is no datapoint that has modified its membership, the time series clustering process is finished. If not the steps from 3 and 5 are to be iterated over again.

DBA iteratively refines an average sequence and follows an expectation-maximization scheme.

Dynamic Time Warping (DTW) algorithm is used to find the distance parameter in time for the given sequence for time series k-means clustering. DTW algorithm can be implemented using the following equations:

$$
\begin{aligned}
& \mathrm{g}(1,1)=\mathrm{d}(1,1) \\
& \mathrm{g}(\mathrm{i}, \mathrm{k})=\min \{\mathrm{g}(\mathrm{i}-1, \mathrm{k}-1)+\mathrm{d}(\mathrm{i}, \mathrm{k}), \\
& \mathrm{g}(\mathrm{i}, \mathrm{k}-1)+\mathrm{d}(\mathrm{i}, \mathrm{k}), \\
& \mathrm{g}(\mathrm{i}-1, \mathrm{k})+\mathrm{d}(\mathrm{i}, \mathrm{k})\}
\end{aligned}
$$

Time Warping Window $\mathrm{k}-\mathrm{r} \leq \mathrm{i} \leq \mathrm{k}+\mathrm{r}$.

Time normalized distance parameter

$$
\mathrm{D}(\mathrm{A}, \mathrm{B})=\mathrm{g}(\mathrm{n}, \mathrm{m}) /(\mathrm{n}+\mathrm{m})
$$


This is the dynamic programming solution to the problem. $g(m, n)$ refers to the value of a point in the distance map. DTW Barycenter Averaging (DBA) hierarchical clustering algorithm is run post applying the dtw distance metric scheme. Clustering results in two separate waveforms of vibration. One that has centroid of the high frequency vibration, i.e., when the machine has gone faulty and the other that has centroid in the time series vibration data where the vibrations are of lesser intensity and can be compared with that which occurs in a healthy machine that is not subject to the wearing off process. Similarly, this algorithm can also be extended for temperature data with a few modifications to the hyperparameters. The scalable light weight model is easily deployed in the on-board controller of the machine system. AI controllers such as the Nvidia Jetson family boards show faster processing and improved result inference timings for this model and are also cheaper to upgrade for the machine system.
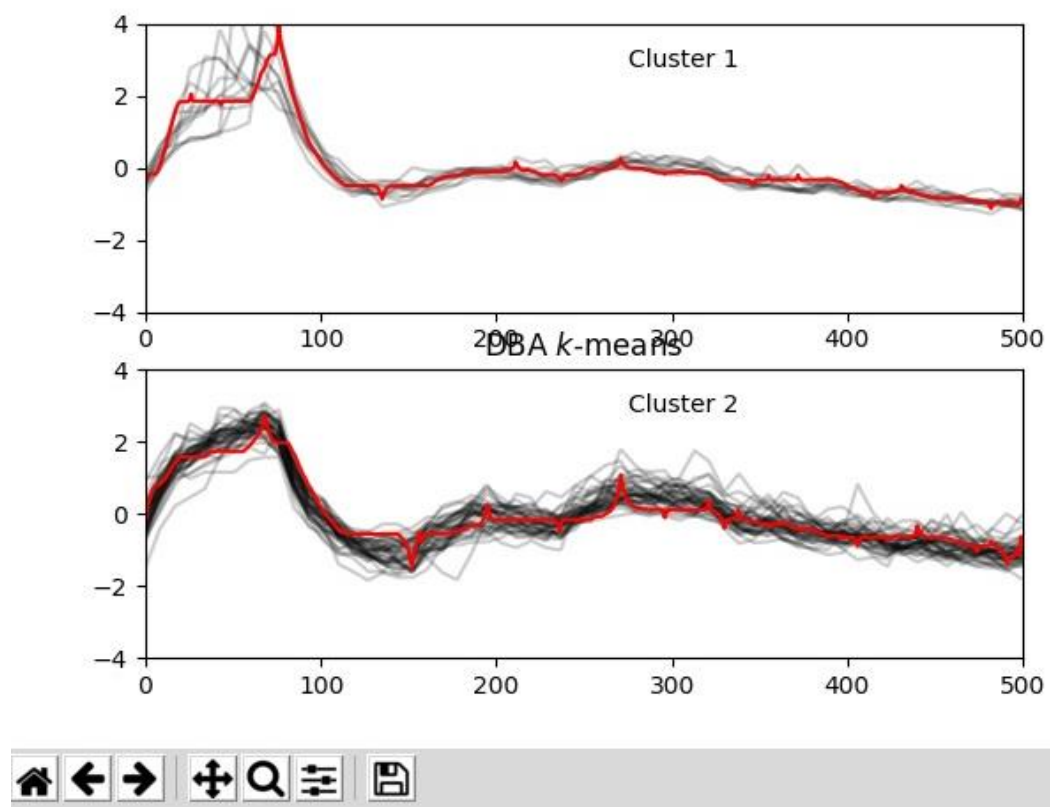

Figure 1 DBA clustering resulting in a segregation between the much vibrating sequence (cluster2faulty) as against the less vibrating sequence cluster 1 (healthy machine) 
Early Maintenance and Diagnosis of Connected Machines Using Machine Learning

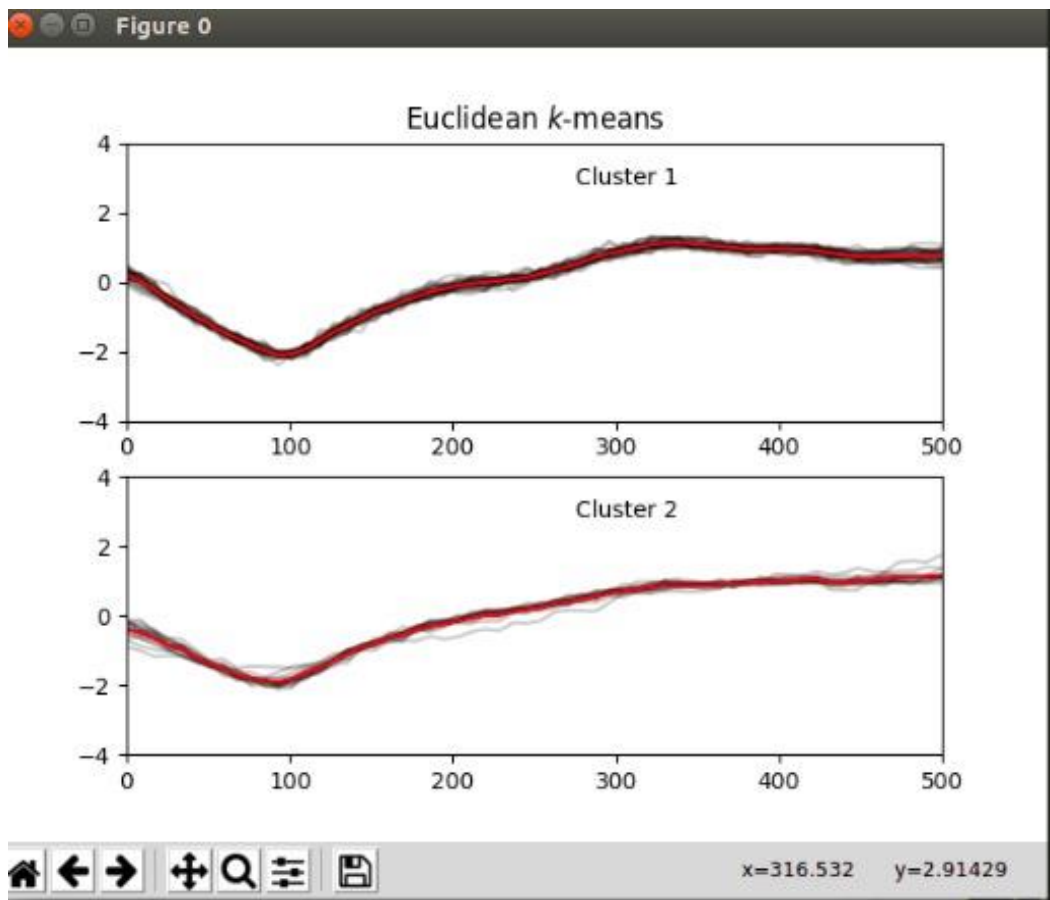

Figure 2 k-means clustered temperature sequence with 50 data points. First graph shows the functional range (range of allowed fluctuations) and the second one shows the defective range (range of defective fluctuations).

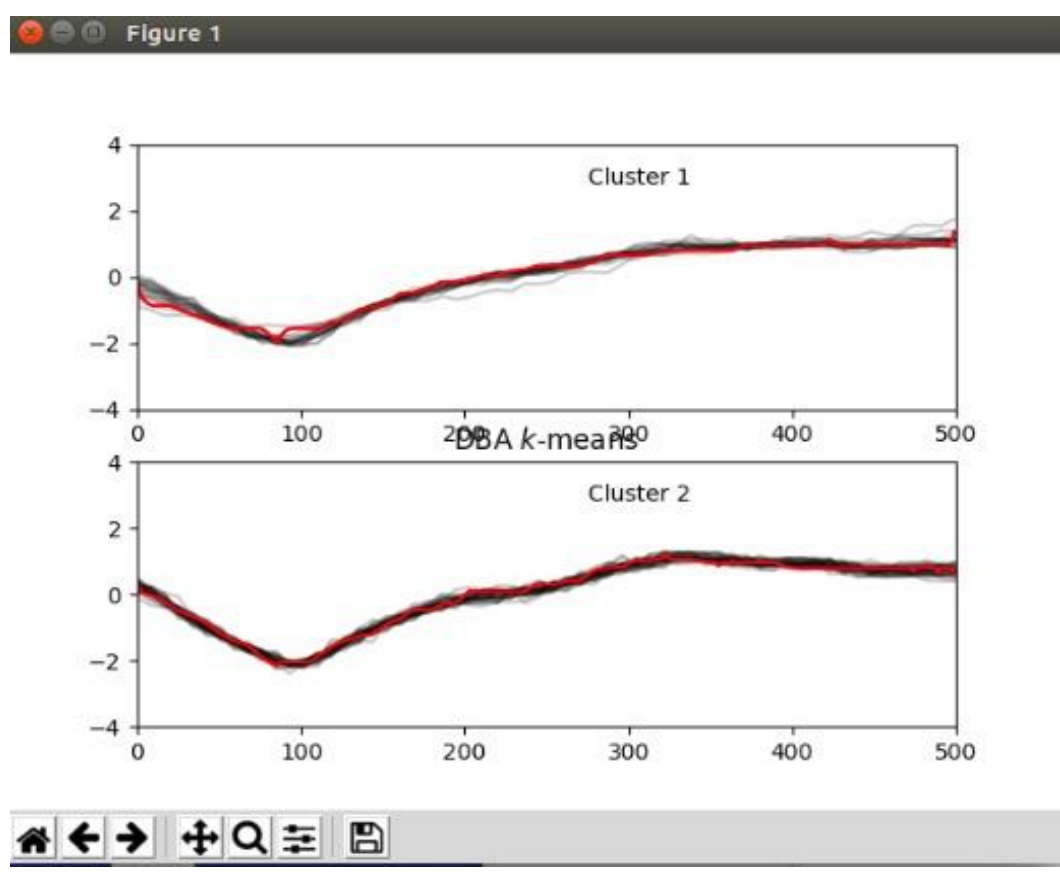

Figure 3 DBA clustered temperature sequence with 50 data points. First graph shows the functional range (range of allowed fluctuations) and the second one shows the defective range (range of defective fluctuations). 


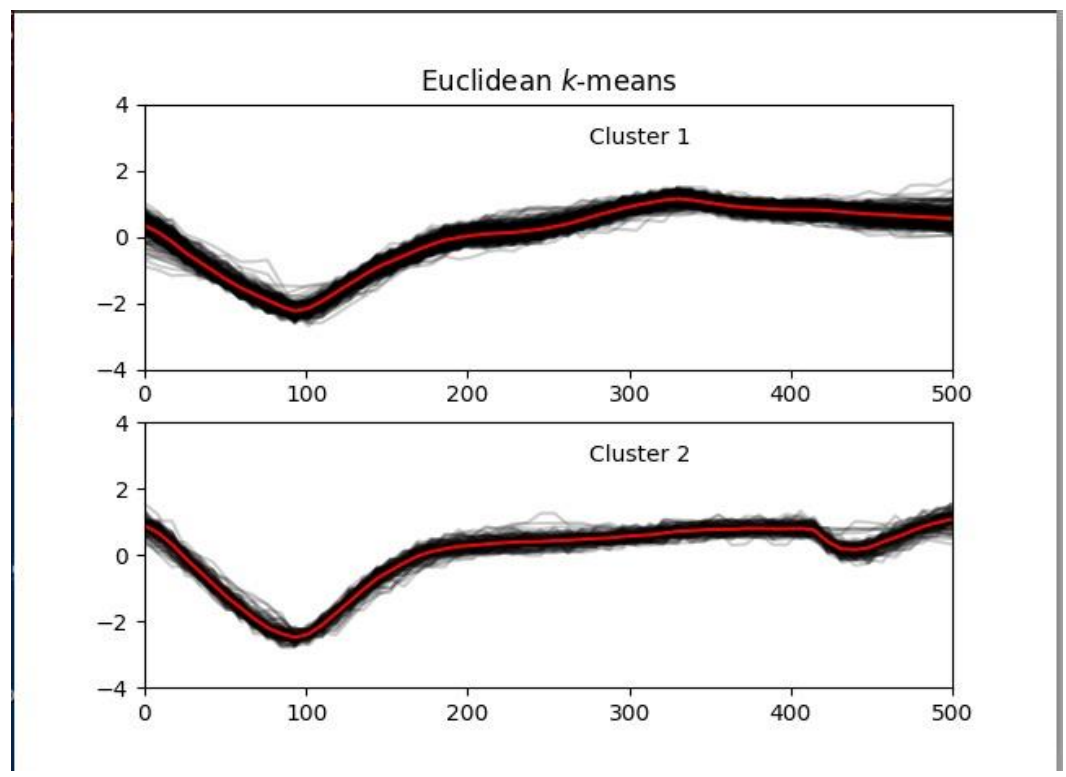

Figure 4 k-means clustered temperature sequence with 500 data points.

\section{$\otimes \Theta($ Figure 0}
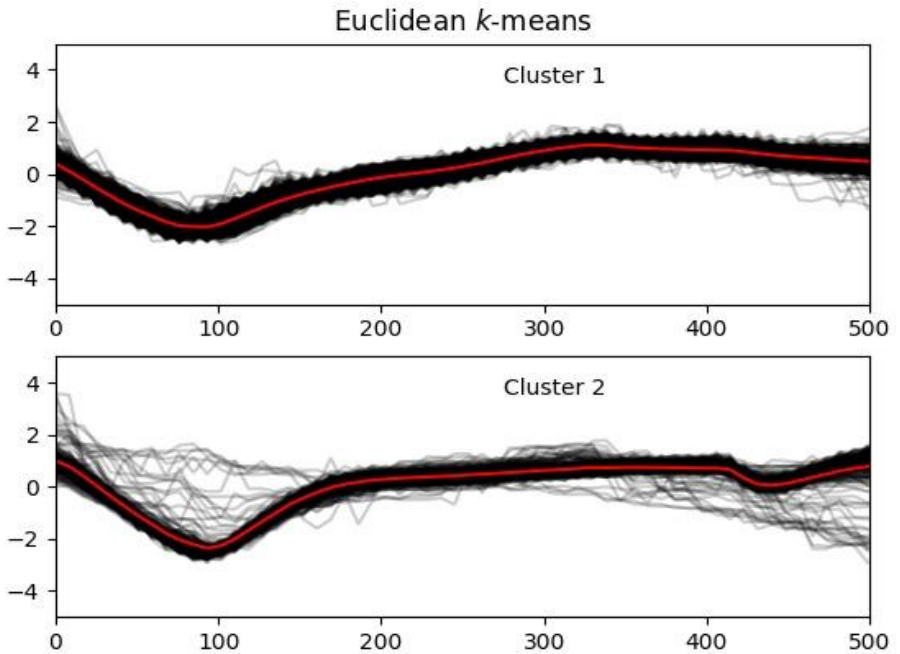

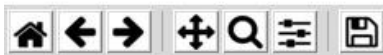

Figure 5 k-means clustered temperature sequence with 2205 data points.

Figure 5 clearly shows temperature fluctuations totally off range so that it causes the machine to dysfunction in the cluster 2 graph.

\section{CONCLUSIONS}

This paper thus brings to light the possible advantages of using a simple yet carefully modified algorithm, the k-means clustering approach along with a more strengthened formula of DBM clustering to understand time series behavior of machine parameters. This is the major step towards converting to a fully machine-driven ecosystem operated autonomously. In the context of autonomous systems, it is important to use the present behavior of the system to predict the health of the machine in a future time instance. This is effectively achieved using the lightweight AI model deployed and demonstrated in this paper. This model is highly scalable to unknown machine parameters that can be evolved in future even for 
machines that haven't been invented yet. Even a quantum computer with its intrinsic complex circuitry and sub normal temperatures of operation would require heavy monitoring of its parameters. This approach promises a load-less way of seamlessly monitoring and reporting faults or impending failure of the machine part to the nearby control center. In a fully automated system, such a report can be directly linked to a fault treatment control system such as to enhance the coolant levels in case of overheating of the component. One of the major concerns however, is the intricacy of using this monitoring system in regions where the sensor/ feedback controller itself cannot be deployed. But such cases are extremely rare as even the riskiest situations such as outer space systems have extensive sensor systems to report the fluctuations of parameters along with a feedback controller to rectify the erroneous situations.

\section{REFERENCES}

[1] Satoh. F, Itakura. M, "Cloud-based Infrastructure for Managing and Analyzing Environmental Resources”, in SRII Global Conference, pp.325-334, 201.

[2] Guangxing Niu, Shijie Tang, Bin Zhang, (2018) "Machine Condition Prediction Based on Long Short Term Memory and Particle Filtering", in IECON 2018 - 44th Annual Conference of the IEEE Industrial Electronics Society, pp. 5942-5947

[3] Fink P.K and Lusth J. (1987), "Expert systems and diagnostic expertise in the mechanical and electrical domains", in IEEE Trans. on Systems, Man and Cybernetics, Vol. SMC-17, No.13, May/Jun.

[4] Frank P.M. and Köppen-Seliger B. (1997), "New Developments Using AI in Fault Diagnosis", in Engg. Applications Art. Intell., Vol. 10, No.1, pp.3-14.

[5] Rambabu K, Misra R.P. and Anand G. (2009), "Justification of world-class maintenance using analytic hierarchy constant sum method", in Journal of Quality in Maintenance Engineering, Vol.15, Iss: 1, pp.47-77.

[6] Christos E., Liyanage J.P. and Jantunen E., (2009), "Mobile solutions for engineering asset and maintenance management", in Journal of Quality in Maintenance Engineering, Vol. 15, Iss.: 1, pp. $92-105$.

[7] "An Industrial Case Study Using Vibration Data and Machine Learning to Predict Asset Health", in Business Informatics (CBI) 2018 IEEE 20th Conference on, vol. 01, pp. 178$185,2018$.

[8] Will Nash, Tom Drummond, Nick Birbilis, (2018) "A review of deep learning in the study of materials degradation", in npj Materials Degradation, vol. 2

[9] F. Hahn, M. Pablo, J. Reyes "Solar Driven Wind Speed Monitoring System Using Wireless or Wired Sensors" in SciRes, Energy and Power Engineering, Vol 6, PP.213221,2014

[10] Jianjing Zhang, Peng Wang, Robert X. Gao, (2018) "Modeling of Layer-wise Additive Manufacturing for Part Quality Prediction", in Procedia Manufacturing, vol. 16, pp. 155.

[11] J. U. Duncombe, (1959) "Infrared navigation-Part I: An assessment of feasibility," in IEEE Trans. Electron Devices, vol. ED-11, pp. 34-39, Jan.

[12] C. Y. Lin, M. Wu, J. A. Bloom, I. J. Cox, and M. Miller, (2001) "Rotation, scale, and translation resilient public watermarking for images," in IEEE Trans. Image Process., vol. 10, no. 5, pp. 767-782, May

[13] C.V.Ravi kumar, Kala Praveen Bagadi, (2016) "Performance analysis of IPv4 to IPv6 Transition Methods." Indian Journal of Science and Technology, may,Vol .9 issue 20,90005 
[14] Nadine Martin, Pierre Granjon and Christine Serviere from the SAIGA research team, The GOTIX project, housed by the GIPSA-lab, aims to characterize the defects on electrically driven mechanical systems. Available: http://www.gipsa-lab.grenoble-inp.fr/projet/gotix/

[15] Castro P., Afonso J.L., Afonso J.A. (2017) "A Low-Cost ZigBee-Based Wireless Industrial Automation System", in Garrido P., Soares F., Moreira A. (eds) CONTROLO 2016. Lecture Notes in Electrical Engineering, vol 402. Springer, Cham.

[16] C.V. Ravikumar, Kala Praveen Bagadi, (2016)" Robust Neural network based multiuser detector in MC-CDMA mMAI mitigation." Indian Journal of Science and Technology, Aug ,Vol. 9 issue 30.95994

[17] Niennattrakul, Vit \& Ratanamahatana, Chotirat. (2007). "On Clustering Multimedia Time Series Data Using K-Means and Dynamic Time Warping" in Multimedia and Ubiquitous Engineering International Conference. 733-738. 10.1109/MUE.2007.165.

[18] Prasanna Tamilselvan, Pingfeng Wang, (2013) "Failure diagnosis using deep belief learning based health state classification", in Reliability Engineering \& System Safety, Volume 115, Pages 124-135, ISSN, 0951-8320, https://doi.org/10.1016/j.ress.2013.02.022.

[19] C.V. Ravikumar, Kala Praveen Bagadi, (2016) "Performance analysis of HSRP in provisioning Layer-3 Gateway Redundancy for Corporate Networks." Indian Journal of Science and Technology, may, Vol. 9 issue 20.89851

[20] Kanaparthy Rama Bramham \& Ravi Kumar CV. (2015) "Comparison and Optimization of Layer2 and Multilayer switch protocols to implement converged and reliable network." International Journal of Applied Engineering and Research, Vol 10,No. 8, pp. 20139-20154.

[21] P.K. Kankar, Satish C. Sharma, S.P. Harsha, (2011) "Fault diagnosis of ball bearings using machine learning methods", in Expert Systems with Applications, Volume 38, Issue 3, Pages 1876-1886, ISSN 0957-4174, https://doi.org/10.1016/j.eswa.2010.07.119.

[22] P. Tamilselvan, Y. Wang and P. Wang, (2012) "Deep Belief Network based state classification for structural health diagnosis," in 2012 IEEE Aerospace Conference, Big Sky, MT, pp. 1-11.

[23] Adnan Nuhic, Tarik Terzimehic, Thomas Soczka-Guth, Michael Buchholz, Klaus Dietmayer, (2013) "Health diagnosis and remaining useful life prognostics of lithium-ion batteries using data-driven methods," in Journal of Power Sources, Volume 239, Pages 680-688, ISSN 0378-7753, https://doi.org/10.1016/j.jpowsour.2012.11.146

[24] Rolf Isermann, (2005) Model-based fault-detection and diagnosis - status and applications, Annual Reviews in Control, Volume 29, Issue 1, Pages 71-85, ISSN 13675788, https://doi.org/10.1016/j.arcontrol.2004.12.002.

[25] M. A. Awadallah and M. M. Morcos, (2006) "Automatic diagnosis and location of openswitch fault in brushless DC motor drives using wavelets and neuro-fuzzy systems," in IEEE Transactions on Energy Conversion, vol. 21, no. 1, pp. 104-111, March

[26] Adam Glowacz, Zygfryd Glowacz, (2017) Diagnosis of the three-phase induction motor using thermal imaging, Infrared Physics \& Technology, Volume 81, Pages 7-16, ISSN 1350-4495, https://doi.org/10.1016/j.infrared.2016.12.003.

[27] Chuan Li, René-Vinicio Sánchez, Grover Zurita, Mariela Cerrada, Diego Cabrera, (2016) "Fault Diagnosis for Rotating Machinery Using Vibration Measurement Deep Statistical Feature Learning", in Sensors, 16, 895, https://doi.org/10.3390/s16060895

[28] C.V. Ravikumar, Kala Praveen Bagadi, (2017) " Receiver design using artificial Neural Network for signal detection in MC-CDMA system." International Journal of Intelligent Engineering \& Systems", Jan 
[29] C.V. Ravikumar, Saranya KC, (2016) " Implementing Mobile adhoc Networks with improved AODV protocol" International Journal of Applied Engineering and Research, Vol 11,No. 9, pp. 6284-6289

[30] Ruqiang Yan, Robert X. Gao, (2005) "An efficient approach to machine health diagnosis based on harmonic wavelet packet transform", Robotics and Computer-Integrated Manufacturing, Volume 21, Issues 4-5, Pages 291-301, ISSN 0736-5845, https://doi.org/10.1016/j.rcim.2004.10.005.

[31] Verstraete, D., Ferrada, A., Droguett, E. L., Meruane, V., \& Modarres, M. (2017). Deep Learning Enabled Fault Diagnosis Using Time-Frequency Image Analysis of Rolling Element Bearings. Hindawi, 2017, ["5067651"]. https://doi.org/10.1155/2017/5067651. 